\title{
Peak to average power ratio reduction for improving the performance of OFDM system
}

\author{
Ankit Tripathi $^{1^{*}}$, Neha Goel ${ }^{2}$ \\ ${ }^{1^{*}}$ Department of Electronics \& Communication, Raj Kumar Goel Institute of Technology Ghaziabad, INDIA \\ ${ }^{2}$ Department of Electronics \& Communication, Raj Kumar Goel Institute of Technology Ghaziabad, INDIA \\ E-mails: tripathiankit10@gmail.com (ankit tripathi ${ }^{1},{ }^{1}$ Corresponding author), 17nehagoel@gmail.com (neha goel)
}

\begin{abstract}
Orthogonal Frequency Division Multiplexing (OFDM) has a high tolerance to multipath signals and is spectrally efficient making it a good candidate for future wireless communication systems. One disadvantage of OFDM is that the peak of the signal can be up to $\mathrm{N}$ times the average power (where $\mathrm{N}$ is the number of carriers). These large peaks increase the amount of intermodulation distortion resulting in an increase in the error rate. The average signal power must be kept low in order to prevent the transmitter amplifier limiting. Minimizing the PAPR allows a higher average power to be transmitted for a fixed peak power, improving the overall signal to noise ratio at the receiver. It is therefore important to minimize the PAPR. The PAPR of an OFDM signal can be reduced in several ways. In this paper, a technique is described for a better than $5 \mathrm{~dB}$ reduction in the Peak to Average Power Ratio (PAPR) of an OFDM signal. The optimal amplitude and phase of additional peak reduction carriers (PRC) is obtained using a code-book, which is obtained using a search of all possible signal combinations.
\end{abstract}

Keywords: Introduction, Peak Reduction Carriers, Effect of PRC Position, Simulation Results.

DOI: http://dx.doi.org/10.4314/ijest.v4i1.12S

\section{Introduction}

Orthogonal Frequency Division Multiplexing (OFDM) is a multicarrier transmission technique which is widely adopted in different communication applications. OFDM prevents Inter Symbol Interference (ISI) by inserting a guard interval and mitigates the frequency selectivity of a multi-path channel by using a simple equalizer. This simplifies the design of the receiver and leads to inexpensive hardware implementations. Moreover, OFDM offers some advantages in higher order modulations and in the networking operation that position OFDM as the technique of choice for the next generation of wireless networks. However, OFDM systems have the undesirable feature of a large Peak to Average Power Ratio (PAPR) of the transmitted signals.

OFDM is similar to FDMA in that the multiple user access is achieved by subdividing the available bandwidth into multiple channels that are then allocated to users. However, OFDM uses the spectrum much more efficiently by spacing the channels much closer together. This is achieved by making all the carriers orthogonal to one another, preventing interference between the closely spaced carriers. One disadvantage of OFDM is that the peak of the signal can be up to $\mathrm{N}$ times the average power (where $\mathrm{N}$ is the number of carriers). These large peaks increase the amount of inter-modulation distortion resulting in an increase in the error rate. The average signal power must be kept low in order to prevent the transmitter amplifier limiting. Minimizing the PAPR allows a higher average power to be transmitted for a fixed peak power, improving the overall signal to noise ratio at the receiver. It is therefore important to minimize the PAPR. This paper presents a method for reducing the PAPR of a OFDM signal that contains data. It is optimized for a low number of carriers making it a suitable technique for multi-user OFDM. 


\section{Peak Reduction Carrier}

This paper presents a technique that combines selective mapping and cyclic coding. A reduction in the PAPR is achieved by adding extra carriers referred to as Peak Reduction Carriers (PRC). The phase and amplitude of the PRCs is varied to minimize the overall PAPR. The original information carriers are unaffected and can be decoded normally. The receiver can disregard the PRCs, or they can be used for error detection. The frequency of PRCs or relative positioning of the PRCs can be varied with respect to the information carriers depending on the application. The results presented were found using a computationally intensive exhaustive search to find the optimal setting for the PRCs. However it is assumed that further work will allow a more efficient algorithm to be found.

An optimal setting for the PRCs corresponds to the combination of phase and amplitude that achieves the lowest PAPR of the overall OFDM symbol (information carriers and PRCs). In this paper the phase and amplitude of the PRCs was set in a coarse quantized manner to minimize the number of combinations needed to be searched. The phase of the PRCs was set to $0^{\circ}$ or $180^{\circ}$ and the carriers were turned on or off. There are therefore $3^{\mathrm{M}}$ combinations for the PRCs for each information code word (where $\mathrm{M}$ is the number of PRCs). This level of quantization was found to be appropriate for BPSK information carriers. Finer quantization may produce improved results for higher modulation schemes.

An exhaustive search of all combinations of allowable phase and amplitude gives optimal PRCs, but is computationally intensive. This method can be used for small numbers of carriers where the optimal PRC coding can be stored in a look up table or code-book. This is impractical for more than 16 information carriers or for more than 10 PRCs as the number of combinations becomes too large to store and calculate. However for some multi-user OFDM applications 16 or less carriers per user is sufficient. The results shown were calculated based on all combinations of information code words, thus will give a good indication of the practical PAPR improvement.

For each experiment the inverse fast Fourier transform (IFFT) of the carrier configuration was used to give a complex base band signal. Let the complex base band signal be defined as in equation 1.

$$
s(t)=s_{I}(t)+j \cdot s_{Q}(t)
$$

When this is quadrature modulated to RF the signal can be written in polar form as:

$$
S_{\text {rf }}(t)=a(t) \cdot \cos \left(2 \pi \cdot f_{c}(t)+\theta(t)\right)
$$

Where $a(t)$ is the amplitude and $q(t)$ is the phase of the signal. Thus:

$$
a(t)=\sqrt{ }\left(S_{I}^{2}(t)+S_{Q}^{2}(t)\right)
$$

The definition of the PAPR in eqn 4, where T is the OFDM symbol period, can be used for RF as well as base band (Bauml et al, Electronic Letters, 1996).

$$
P A P R_{d B}=10 \log _{10}\left(\frac{\frac{M a x}{0<t<T}\left(a^{2}(t)\right)}{\frac{M e a n}{0<t<T}\left(a^{2}(t)\right)}\right)
$$

For the simulations carried out, the base band carriers were centered on DC and the size of the IFFT was made at least 8 times greater than the number of carriers, oversampling the time domain signal. This ensures that peaks in the signal were accurately represented to get an accurate PAPR (Tellambura,C et al, Electronic Letters, 1997)(Tellambura,C et al, Electronic Letters, 1998). 


\section{Effect of PRC Position}

Previous results are shown for grouped PRCs that were positioned immediately after the data carriers as shown in Figure 1a. Two different positioning tests were performed. One test kept the PRCs grouped together as in Figure 1a, however they were moved with respect to the data carriers as in Figure 1b.The second test positioned the PRCs in a spread out manner. The best spread pattern was established using a randomized search.

Adding PRCs use a significant amount of additional bandwidth. It is therefore important to minimize the number used, or to position the PRCs so that the bandwidth can be reused. For example, in a multi-user OFDM system where each user transmits a block of carriers, the PRCs can be overlapped, i.e. they are transmitted at the same frequency, effectively halving the bandwidth.

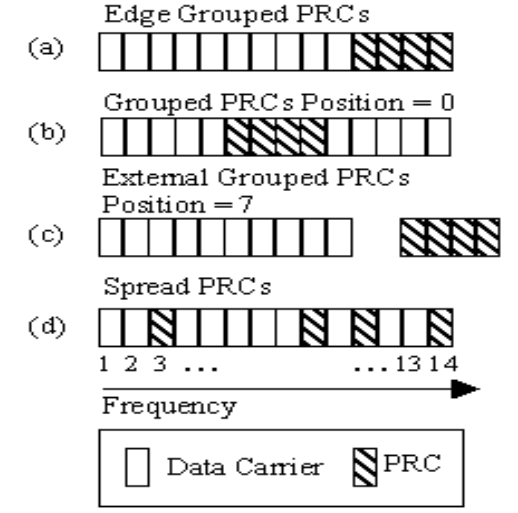

Fig. 2. A three layer artificial neural network [22]

Fig. 1. PRC position combinations

\subsection{Grouped PRCs}

In this scheme the PRCs were maintained as a group of carriers. They were repositioned by sliding them with respect to the data carriers. Figure 2 and 3 show the effect of the position on effectiveness of the PRCs. Figure 5 shows for a small number of PRCs the position of the PRCs within the data carriers has little effect on the performance. However with 4 or more PRCs the position has a significant effect on the performance of the PRCs. Placing the PRCs within the data carriers with an off centre of 3 carriers gives the best results. This gives a further reduction of $1 \mathrm{~dB}$ as compared with edge grouped PRCs. Not having edge grouped PRCs would prevent overlapping of the PRCs for a multi-user OFDM, thus doubling the bandwidth used by the PRCs.

\subsection{Spread PRCs}

The position of the 4 grouped PRCs had a significant effect on the PRC performance, thus it seemed likely that spreading the PRCs out might lead to further improvements. The exact relationship between the position of the PRCs and the PAPR distribution is currently unknown and so a random search was used for optimization. The PRCs and data carriers were positioned randomly to form a block of carriers with no gaps as shown in figure 1d. For each position combination the PAPR distribution was found and the combination which resulted in the lowest maximum PAPR was selected as the optimized PRCs position.

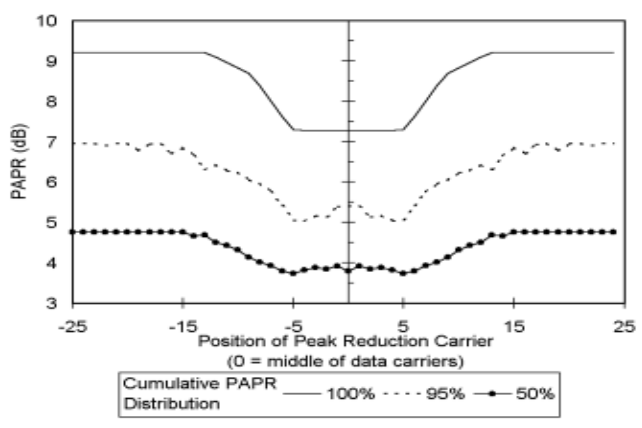

Fig. 2. PAPR verses position of 2 grouped PRCs 


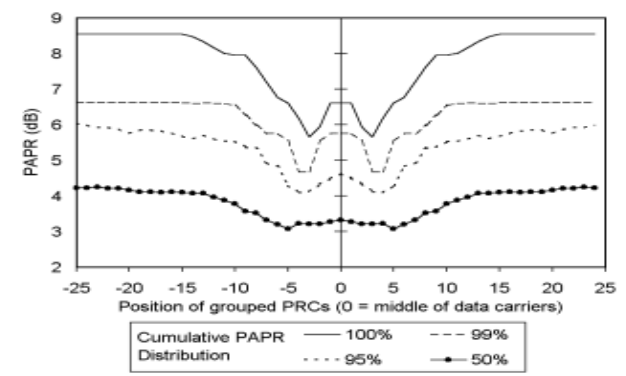

Fig. 3. PAPR verses position of 4 grouped PRCs (10 BPSK data carriers)

The PAPR distribution was found by testing all combinations of the data code words. For each data code word combination the optimum PRCs were found as described in section 3. The PAPR distribution verses the number of PRCs is shown in figure 4. This result is for 10 data carriers and shows that spreading the PRCs can result in large reductions in the PAPR of the OFDM symbol. A reduction of $>6 \mathrm{~dB}$ is possible.

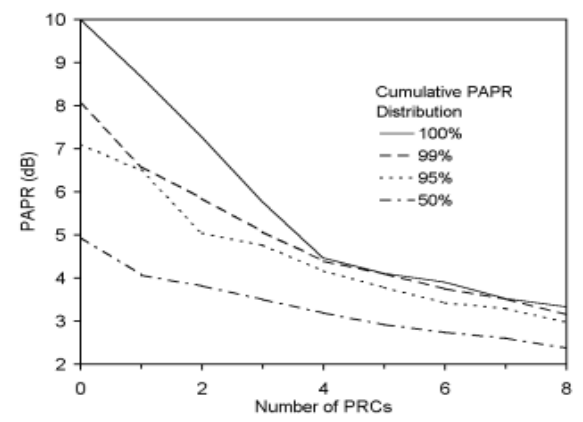

Fig. 4. PAPR verses the number of spread PRCs (10 BPSK data carriers)

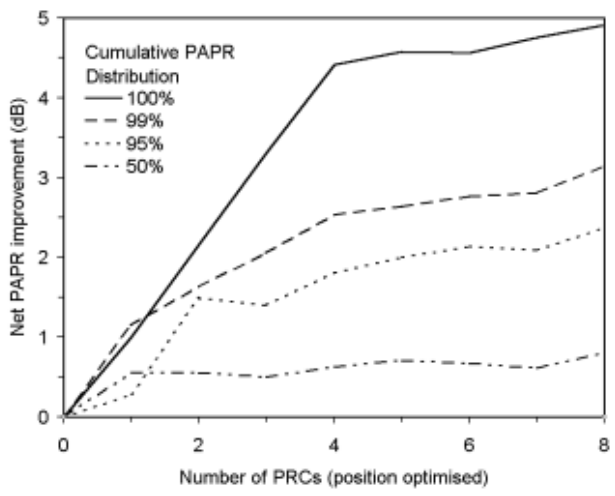

Fig. 5. Net improvement in PAPR, position optimized PRCs (10 BPSK data carriers)

Figure 5 shows the overall net improvement in the PAPR using position optimized PRC. This can be directly compared to figure 7 which shows the results for edge grouped PRCs. The maximum net gain for position optimized PRCs is nearly double that of the edge grouped PRCs. Figure 5 shows that the net PAPR gain increases rapidly up to 4 PRCs, after which the gain is minimal. Thus the optimal number of PRCs would be 4 for 10 data carriers. Other tests also show that the number of PRCs needs to be approximately $40 \%$ of the number of data carriers in order to get significant improvements in the PAPR.

Overlapping of the spread PRCs in a multi-user OFDM system is more difficult as most of the PRCs will be bounded with data carriers. Thus simple overlapping may not be possible. This would be the case if the data and PRC are grouped. However if the carriers for each user are spread out it might be possible have spread out PRCs that overlap between the users, but still provide a large reduction in the PAPR 
Table 1. Shows the number PRC positions tested, and the best combination found.

\begin{tabular}{|l|l|l|l|}
\hline $\begin{array}{l}\text { No. } \\
\text { FRCs }\end{array}$ & $\begin{array}{l}\text { Position } \\
\text { tests } \\
\text { simulated }\end{array}$ & $\begin{array}{l}\text { Fossible } \\
\text { position } \\
\text { combinations }\end{array}$ & $\begin{array}{l}\text { Best Position } \\
\text { Found, see fig. } 4 d \\
\text { for label method }\end{array}$ \\
\hline 1 & 11 & 11 & 1 \\
\hline 2 & 66 & 66 & 2,3 \\
\hline 3 & 286 & 286 & $3,4,6$ \\
\hline 4 & 262 & 1001 & $4,10,12,13$ \\
\hline 5 & 156 & 3003 & $1,3,7,10,15$ \\
\hline 6 & 68 & 8008 & $1,2,7,10,14,16$ \\
\hline 7 & 36 & 19448 & $1,2,7,9,11,14,17$ \\
\hline 8 & 23 & 43758 & $1,2,3,4,11,12,16,18$ \\
\hline
\end{tabular}

The addition of 4 PRCs with 10 data carriers results is a large net gain of $4.5 \mathrm{~dB}$, allowing more power to be transmitted. For a transmission with no PRCs at an error rate of $1 \times 10^{-3}$, adding the PRCs and maintaining the same peak power the error rate would be decreased to $1 \times 10^{-7}$ (Sklar,B, second edition, Prentice Hall, 1988). This is more efficient than adding error correcting bits at the same coding rate. For example using Hamming coding at a rate of 4 parity bits for 11 data bits gives a gain of only $1.2 \mathrm{~dB}$ at a bit error rate of $1 \times 10^{-4}$ which is significantly less than $4.5 \mathrm{~dB}$.

\section{Simulation Results}

The simplest arrangement for the relative positioning of the data and PRCs is to have a block of data carriers immediately followed by a block of PRCs. This arrangement was used for the results shown in Figures6-7

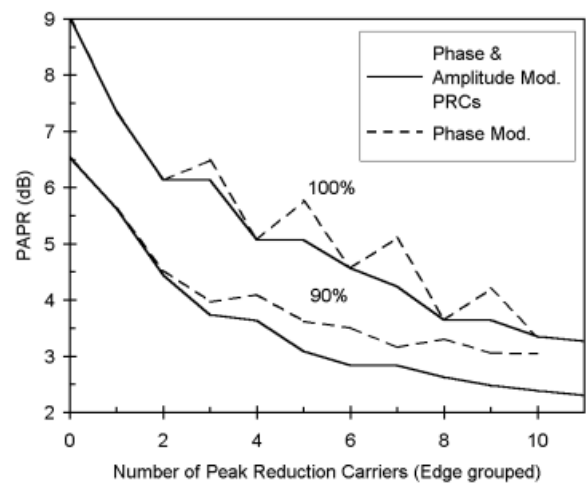

Fig. 6. PAPR verses number of edge grouped PRCs (8 BPSK data carriers)

Figure 6 shows the worst case PAPR and the 90\% point in the cumulative distribution of PAPR as the number of PRCs is increased. The maximum PAPR for the 8 information carriers is reduced by $>5.5 \mathrm{~dB}$ for the addition of 10 PRCs. Selecting the optimal amplitude and phase of the PRC improves the performance significantly as compared with only setting the phase as used in cyclic coding (Wulich et al, Electronic Letters, 1996). For this reason phase and amplitude modulation of the PRCs was used in all later experiments due to the improved performance.

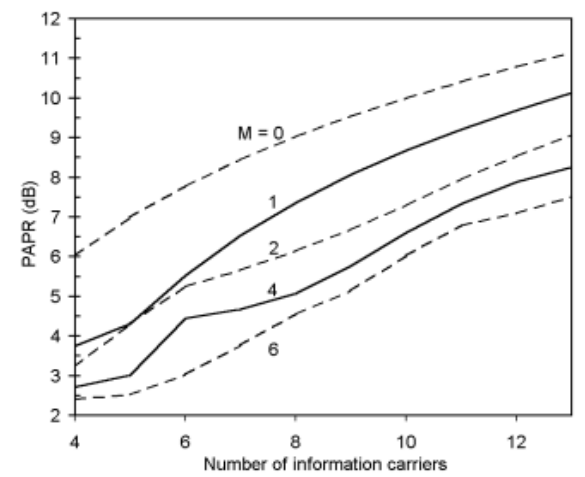

Fig. 7. Maximum PAPR verses number of data carriers and edge grouped PRCs, where $M$ is the number of PRCs. 
Figure 7 shows the effect of adding PRCs to the PAPR as the number of information carriers is varied. The improvement in PAPR remains relatively constant as the number of information carriers is increased. This shows that this technique gives consistent performance gains as the number of information carriers is varied. Adding PRCs reduces the PAPR at the expense of additional transmission power. Figure 7 shows the net improvement in PAPR due to the addition of PRCs. The PAPR reduction was calculated as the difference between the PAPR results for zero PRCs and the PAPR results with the addition of PRCs. The loss in signal power due to the PRCs was then subtracted from the PAPR reduction in order to give the net PAPR improvement. If the data signal power lost due to the transmission of the PRCs was more than the PAPR gain then there would be little point in adding the PRCs. It can be seen that for 10 BPSK carriers there is little improvement in adding more than 2 PRCs. In fact adding more than 5 PRCs results in a worsening of the average (50\%) PAPR. This is due to the power cost of the PRCs.

\section{Conclusion}

From the above graphs, we have found that the PRCs reduction of $>6 \mathrm{~dB}$ in the maximum PAPR and a net reduction of $>4.5 \mathrm{~dB}$ when the additional power for PRCs is taken into account. It was found that varying the amplitude as well as phase for the PRCs gave improved performance over just phase variation. It was also found that spreading the position of the PRCs resulted in a better performance than grouped PRCs. Adding more PRCs results in a lower PAPR, however the use of large numbers of PRCs is limited by the cost of additional transmission power, bandwidth and complexity limits.

\section{References}

Bauml, R.W., Fischer, R.F.H., Huber, J.B., Reducing the peak-to-average power ratio of multicarrier modulation by selected mapping, Electronic Letters, 1996, Vol. 32, pp. 2056-2057

Van Eetvelt, J., Wade, G., Tomlinson, M., Peak to average power reduction for OFDM schemes by selective scrambling, Electronic Letters, 1996, Vol. 32, pp. 1963-1964

Wulich, D., Reduction of peak to mean ratio of multicarrier modulation using cyclic coding, Electronic Letters, 1996, Vol. 32, pp. 432-433

Tellambura, C., Use of m-sequences for OFDM peak-to-average power ratio reduction, Electronic. Letters, 1997, Vol. 33, pp. 1300-1301

Tellambura, C., Phase optimization criterion for reducing peak-to-average power ratio in OFDM, Electronic Letters, 1998, Vol. 34, pp. 169-170

Davis, J.A., Jedwab, J., Peak-to-mean power control and error correction for OFDM transmission using Golay sequences and Reed Muller codes, Electronic Letters, 1997, Vol. 33, pp. 267-268

Skylar, B., Digital Communications Fundamentals and Applications, Prentice Hall, 1988, pp. 300

\section{Biographies}

Ankit Tripathi received M.Tech from Kamla Nehru Institute of Technology Sultanpur, India in 2011 \& B.Tech (Hons.) from IET government college of Dr. R.M.L awadh University Faizabad, India in 2007. He is an Assistant Professor in the department of Electronics \& Communication Engineering., Raj Kumar Goel Institute of Technology, India. He is a fellow of ICEIT (India) \& CSTA.He has Presented \& Published more than 12 papers in National \& International Conferences. He has also published one paper in International Journal. His research interests include Digital Communication, Mobile Communication \& Orthogonal Frequency Division Multiplexing systems.

Neha Goel is Pursuing PhD from SRM University Chennai, India. She is an Assistant Professor in the department of Electronics \& Communication Engineering, Raj Kumar Goel Institute of Technology, India. She has Seven years of experience in Teaching. She is a fellow of ISCA (India), ICEIT (India) \& CSTA. She has Presented \& Published more than 12 papers in National \& International Conferences. She has also published one paper in International Journal. Her research interests Digital Systems, Digital CMOS Design.

Received January 2012

Accepted February 2012

Final acceptance in revised form March 2012 